\title{
Clusterizando Mapas Conceituais para Identificar Desempenho Cognitivo em Grupos
}

\author{
Marcos P. D. Lovati' ${ }^{1}$, Camila Z. Aguiar', Ramon A. Azeredo', Davidson Cury ${ }^{1}$ \\ ${ }^{1}$ Departamento de Informática - Universidade Federal do Espírito Santo (UFES) \\ Goiabeiras - 29.000-000 - Vitória - ES - Brasil \\ \{marcos.lovati, camila.zacche.aguiar, ramon.ahnert, dedecury\}@gmail.com
}

\begin{abstract}
Collaborative work is a very useful teaching-learning strategy to encourage social interactions among learners of different expectations and perspectives of knowledge, provoking greater motivation to solve problems. The present work proposes a tool for clustering concept maps with the objective of assisting the teacher in the organization of groups of learners based on their cognitive performance. From them, homogeneous and heterogeneous groups can be formed to provide adequate instrumental material and apply different pedagogical strategies.
\end{abstract}

Resumo. Trabalhos colaborativos constituem uma estratégia de ensino muito útil ao incentivar interações sociais entre aprendizes de diferentes expectativas e perspectivas de conhecimento, provocando maior motivação para a resolução de problemas. Nesse sentido, o presente trabalho propõe uma ferramenta de clusterização de mapas conceituais com o objetivo de auxiliar o professor na organização de grupos de aprendizes com base no seu desempenho cognitivo. A partir dela, grupos homogêneos e heterogêneos podem ser formados para fornecimento de material instrucional adequado e aplicação de diferentes estratégias pedagógicas.

\section{Introdução}

Trabalhos colaborativos constituem uma estratégia de ensino-aprendizagem muito útil ao incentivar as interações sociais entre aprendizes. Na colaboração, ideias opostas ou ao menos diferentes podem ser colocadas num espaço comum, o que incentiva a participação e contribui para o desenvolvimento e a construção de novos conhecimentos [Moro 1991]. Assim, para melhorar as condições de interação, é desejável que o professor consiga organizar grupos de aprendizes com diferentes expectativas e perspectivas de conhecimento [Wood e O'Malley 1996].

Nesse contexto, estabelecer critérios para a formação de grupos pode favorecer a dinâmica e a qualidade da aprendizagem coletiva. Pontos de vista que se contrastam, vocabulários desenvolvidos e deficientes, aprendizes novatos e veteranos são alguns dos critérios possíveis de serem analisados e utilizados. Contudo, a tarefa de formar grupos potenciais pode desprender um trabalho complexo e custoso para o professor caso não seja utilizada uma ferramenta computacional adequada.

Uma vez que foram criados para organizar e representar o conhecimento 
individual ou coletivo, os mapas conceituais se tornaram também ferramentas úteis para demonstrar o desempenho cognitivo de cada aprendiz. Com base nessa premissa, o presente trabalho propõe uma ferramenta de clusterização de mapas conceituais para apoiar o professor na tarefa de organizar aprendizes em grupos, tendo por base o desempenho cognitivo neles observado. Ao permitir o agrupamento de aprendizes com mapas semelhantes ou divergentes, a ferramenta auxilia na redução do esforço desprendido pelo professor em direcionar material instrucional significativo a cada aprendiz e formar grupos heterogêneos para a realização de trabalhos colaborativos. A proposta é apresentada em forma de um protótipo e os resultados obtidos são criticamente analisados.

Apresentamos, na Seção 2, as interações sociais e a aprendizagem com mapas conceituais; na Seção 3, as técnicas propostas para avaliação de mapas; na Seção 4, a proposta da ferramenta de clusterização de mapas para a organização de grupos; na Seção 5, o experimento realizado a partir do protótipo e, por fim, na Seção 6, as conclusões obtidas e os trabalhos futuros.

\section{Interações Sociais e a Aprendizagem com Mapas Conceituais}

Segundo [Vygotsky 2007], as interações sociais são o meio sobre o qual as crianças adentram na vida intelectual umas das outras. Os processos de desenvolvimento iniciados pela aprendizagem são executados somente quando as crianças interagem entre si e com outras pessoas mais experientes, por meio de tarefas cooperativas. Nesse sentido, [Davis et al. 1989] afirmam que as relações que se estabelecem durante o processo de educação e ensino são tão importantes para o desenvolvimento cognitivo quanto o conteúdo a ser apropriado.

Quando os grupos de trabalho são formados por membros de diferentes expectativas e perspectivas, é provável que a elaboração do conhecimento e a motivação para resolução de problemas ocorram mais acentuadamente [Wood e O’Malley 1996]. A aprendizagem e o desenvolvimento são produto do confronto de diferentes ideias, o que ocorre mais frequentemente em grupos heterogêneos. Dessa forma, os conflitos agem como impulsionadores de substanciais avanços cognitivos [Moro 1991].

Portanto, identificar os diferentes perfis existentes entre os aprendizes de uma turma e incentivar o constante diálogo entre eles possui grande importância para o seu desenvolvimento cognitivo. Nesse contexto, os mapas conceituais construídos por aprendizes podem ser utilizados para a identificação desses diferentes perfis.

Os mapas conceituais são ferramentas gráficas formadas basicamente por conceitos e suas relações, criados com intuito de representar e organizar o conhecimento [Novak 1977]. Tais conceitos e relações são dispostos em forma de triplas (conceitorelação-conceito), de modo a formar proposições significativas [Cañas et al. 2006]. Dos mapas conceituais podem ser feitos diversos usos, desde o apoio à aprendizagem até a sua análise e avaliação [Mintzes et al. 2000].

Por meio dos mapas construídos pelos aprendizes, o professor poderá verificar como cada um "estrutura, hierarquiza, diferencia, relaciona, discrimina e integra conceitos de determinada unidade de estudo, tópico, disciplina etc." [Moreira 1984]. Por 
isso, o conhecimento assim representado pode ser analisado sob diversos aspectos, que vão desde a disposição espacial dos conceitos no mapa até a validade das proposições formadas. O resultado da análise individual pode indicar o desempenho cognitivo do aprendiz e auxiliar o professor no sequenciamento das tarefas de aprendizagem a serem aplicadas [Novak e Cañas 2010].

Como resultado de uma análise coletiva de mapas, espera-se que o professor possa identificar aprendizes que possuem desempenho cognitivo semelhante, objetivando fornecer-lhes o mesmo material instrucional. Ao identificar aprendizes com desempenhos cognitivos diferentes, o professor poderá agrupá-los para realização de atividades colaborativas e assim fazer com que se beneficiem da troca de conhecimentos, tal como fundamentam os autores citados anteriormente.

A seguir, são apresentadas algumas abordagens que utilizam diferentes aspectos dos mapas conceituais com a finalidade de otimizar a formação de grupos.

\section{Avaliação de Mapas Conceituais}

Várias técnicas têm sido propostas com o objetivo de permitir a avaliação da aprendizagem por meio de mapas conceituais e viabilizar a utilização de certas estratégias pedagógicas, algumas delas com a utilização de ferramentas computacionais. Considerando que a análise do conhecimento construído por cada aprendiz de maneira individual não oferece meios diretos para a aplicação de estratégias pedagógicas adequadas a grupos, algumas estratégias de classificação de mapas baseadas em critérios foram propostas com o intuito de permitir o agrupamento dos seus autores.

[Kinchin e Hay 2004] elegeram como critério de classificação o tipo de aspecto morfológico ("spoke", "chain" ou "net") dos mapas conceituais construídos pelos aprendizes. O método usado para a análise foi a aparência visual, não sendo utilizada qualquer ferramenta computacional. A proposta incentiva a formação de grupos com integrantes que apresentam estruturas distintas de mapas para certo tópico, considerando que elas indicam diferentes perspectivas sobre o mesmo assunto que, juntas, podem incentivar a construção coletiva de conhecimento. Porém, por não utilizar nenhum parâmetro mensurável, a classificação dos mapas em um dos tipos pode ser subjetiva e variar de avaliador para avaliador, como afirmam os autores.

[Valerio et al. 2008] apresentaram a construção e validação de uma ferramenta capaz de classificar automaticamente mapas conceituais baseando-se na taxonomia topológica de sete níveis concebida por [Cañas et al. 2006]. Nessa abordagem, cada mapa é avaliado segundo cinco critérios conhecidos por serem bons descritores de estrutura em mapas conceituais. Os testes realizados com a ferramenta constataram que mapas de especialistas puderam ser classificados em níveis superiores aos de aprendizes. Contudo, não há indicação sobre o quanto mapas de mesmo nível são semelhantes em termos de conteúdo, nem quantos conceitos são compartilhados por mapas de diferentes níveis, o que seria valioso para a composição de grupos homogêneos e heterogêneos.

[Kardan et al. 2009] criaram um sistema de ranqueamento de mapas com o objetivo de identificar especialistas dentre um grupo de autores. $\mathrm{O}$ primeiro passo 
consiste em comparar os conceitos existentes nos mapas (tags) com as palavras-chave de documentos relevantes para o assunto. Depois, os mapas são avaliados e classificados utilizando a ferramenta criada por [Valerio et al. 2008]. Os resultados das duas etapas são combinados para verificar a qualidade do conhecimento representado e assim inferir quais autores são especialistas, para que eles sejam colocados em grupos distintos para realização de atividades colaborativas. Todavia, a proposta não deixa claro se seria possível organizar a turma como um todo com base nos níveis de desempenho identificados.

As abordagens encontradas a partir da revisão da literatura reafirmam a importância de identificar os aprendizes com diferentes perfis e tentar organizá-los para melhorar as condições da aprendizagem coletiva. Contudo, dentre elas, não foi encontrada nenhuma que leve em conta a semelhança de conteúdo (conceitos e proposições) entre os mapas como critério de agrupamento dos aprendizes. Nesse contexto, levanta-se a hipótese de que a similaridade possa ser usada como critério de classificação de mapas e facilitar a organização de grupos de aprendizes para posterior aplicação de estratégias pedagógicas. A seguir, é apresentada uma proposta de ferramenta inerente a essa questão.

\section{Proposta de Ferramenta}

A clusterização é um processo não supervisionado de classificação que tem como meta descobrir naturais agrupamentos entre um conjunto de padrões, pontos ou objetos [Jain 2010] comumente aplicado a bases documentais. Os algoritmos usados neste processo acessam somente as informações contidas em cada documento para agrupá-los segundo alguma métrica de similaridade, não havendo qualquer informação inicial sobre onde os documentos devem ser colocados [Wagstaff et al. 2001].

Assim, considera-se expandir essa abordagem aplicada a bases documentais também para bases de mapas conceituais. A principal diferença observada entre essas tarefas é que a clusterização de documentos geralmente é aplicada sobre uma base com textos de domínios ou assuntos diferentes, nos quais a co-ocorrência de termos (ou conceitos) entre documentos de grupos diferentes é mais rara, o que proporciona uma melhor qualidade de clusterização. Considerando que os mapas serão do mesmo domínio e assunto (onde a co-ocorrência de conceitos entre mapas é maior), e com o objetivo de proporcionar uma análise de melhor qualidade sobre o desempenho cognitivo dos aprendizes, a ferramenta proposta leva em conta não somente os conceitos de forma isolada, mas também a existência de relação entre dois conceitos e as proposições completas.

A seguir é apresentado o modelo conceitual da ferramenta proposta para clusterização de mapas conceituais em fase prototípica que visa o agrupamento de mapas segundo sua similaridade. A ferramenta foi desenvolvida na linguagem Python e dividida em quatro módulos, a saber: Extração de Informação, Processamento Textual, Clusterização e Mediador. A Figura 1 demonstra uma síntese do seu modelo conceitual. 


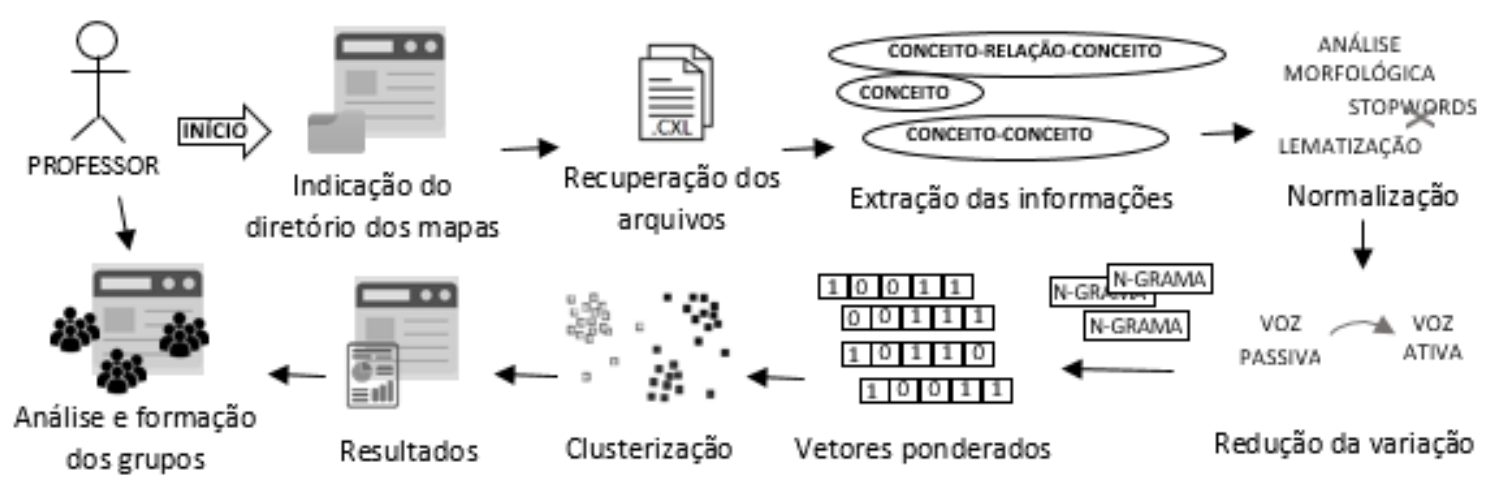

Figura 1. Síntese do modelo conceitual da ferramenta

\subsection{Módulo de Extração de Informações}

O módulo de extração de informações realiza duas atividades: (i) recuperação dos mapas conceituais em formato do CMapTools ${ }^{1}$ (.cxl) no diretório indicado no módulo do mediador e (ii) extração dos três tipos de n-gramas a serem usados pelos módulos seguintes utilizando a biblioteca BeautifulSoap ${ }^{2}$ : n-gramas conceito, composto pelos conceitos do mapa; n-gramas conceito-conceito, composto pelos pares de conceitos que possuem qualquer tipo de ligação; e n-gramas conceito-relação-conceito, composto pelas proposições.

\subsection{Módulo de Processamento Textual}

O módulo de processamento textual tem a função de preparar os n-gramas extraídos pelo módulo anterior para ser processado pelo algoritmo de clusterização, utilizando, para isso, técnicas de Processamento de Linguagem Natural (PLN).

A primeira etapa é a de normalização, compreendendo atividades de: (i) análise morfológica, para identificação da classe gramatical; (ii) remoção de stopwords (palavras com pouca importância significativa); e (iii) lematização (redução da palavra ao seu lema, ou seja, palavra sem flexões). Para a realização dessa etapa, foi utilizada a ferramenta TreeTagger [Schmid 1994], que apresentou melhores resultados na identificação dos lemas em relação a outras testadas, tais como o Lexiconista ${ }^{3}$, LXTagger ${ }^{4}$ e MXPOST ${ }^{5}$ [Ratnaparkhi 1996].

A próxima etapa é a redução da variação. Para isso é realizada a conversão de voz passiva para ativa, ou seja, será invertida a ordem dos conceitos quando as proposições estiverem na voz verbal passiva. Foram definidas algumas regras para identificar proposições em voz passiva que basicamente consideram como tal locuções verbais com verbo de estado seguido de um verbo de ação no particípio (por exemplo, "é representado", "foi realizado", "está definido").

\footnotetext{
${ }^{1}$ http://cmap.ihmc.us/

2 https://www.crummy.com/software/BeautifulSoup/bs4/doc/

$3 \mathrm{http} / /$ www.lexiconista.com/datasets/lemmatization/

${ }^{4} \mathrm{http}$ ///lxcenter.di.fc.ul.pt/tools/en/conteudo/LXTagger.html

5 http://www.inf.ed.ac.uk/resources/nlp/local_doc/MXPOST.html
} 


\subsection{Módulo de Clusterização}

Tomando o conjunto de n-gramas obtido do módulo de processamento textual, o módulo de clusterização representará cada mapa conceitual como um vetor de $n$ dimensões, no qual cada posição se refere a um n-grama ponderado [Souza et al. 2014]. Visto que as estratégias de pesagem que levam em conta a frequência do n-grama no documento não seriam aplicáveis, foi utilizada, inicialmente, a pesagem booleana, atribuindo peso 1 para cada n-grama pertencente ao mapa e peso 0 para não-pertencente.

Em seguida é feita a clusterização propriamente dita. $\mathrm{O}$ algoritmo utilizado foi o algoritmo K-Means da biblioteca Sklearn ${ }^{6}$, que oferece métricas para análise da qualidade da clusterização, tais como a inércia (soma das distâncias dos vetores para o centro de cluster mais próximo) e a média de escore de silhueta (calculada a partir das distâncias entre documentos do mesmo cluster e as distâncias entre documentos de clusters vizinhos). Essa segunda métrica pode assumir um valor que varia de -1 a 1 ; quanto maior o valor, melhor é a divisão entre os clusters formados. Valores negativos indicam erro na divisão, havendo documentos em clusters incorretos.

Sendo $v$ o número de vetores existentes na base, o algoritmo K-Means será executado com parâmetro de número de clusters variando de 2 até $v$-1. Em cada execução, a quantidade de clusters, a inércia e a média de escore de silhueta são registrados para posterior análise no módulo do mediador.

\subsection{Módulo do Mediador}

O módulo do mediador possui uma interface web desenvolvida no framework Django, pela qual o professor pode acessar as funcionalidades do sistema. O processo realizado no módulo pode ser resumido em três etapas: (i) indicação do diretório no qual estão os arquivos de mapas conceituais para processamento, (ii) análise dos resultados obtidos a partir do processamento e escolha da quantidade de clusters a ser utilizada e (iii) criação de grupos de aprendizes para realização dos trabalhos colaborativos.

$\mathrm{Na}$ primeira etapa, após indicar o diretório no qual estão os arquivos de mapas conceituais em formato .cxl, o professor inicia o processamento. Na segunda etapa, após o processamento, o módulo apresenta o número de clusters formados em cada execução do algoritmo no módulo de clusterização e sua respectiva média de escore de silhueta. Para melhor visualização, os resultados são apresentados por ordem decrescente de qualidade; ou seja, as execuções que obtiverem melhor média de escore de silhueta virão primeiro na lista. O professor escolherá uma quantidade de clusters para seguir com a criação dos grupos de aprendizes.

$\mathrm{Na}$ terceira etapa, o professor poderá usar diferentes estratégias para criar grupos de aprendizes para diferentes tipos de atividades. Por exemplo, quando necessitar de grupos mais homogêneos para o fornecimento de material instrucional, o módulo agrupará aprendizes com mapas pertencentes ao mesmo cluster; quando o professor necessitar de grupos mais heterogêneos para realização de debates sobre determinado assunto, o módulo agrupará aprendizes com mapas de clusters diferentes.

\footnotetext{
${ }^{6}$ sklearn.cluster.Kmeans
} 
VI Congresso Brasileiro de Informática na Educação (CBIE 2017)

Anais do XXVIII Simpósio Brasileiro de Informática na Educação (SBIE 2017)

\section{Experimento e Resultados}

A fim de analisar a eficácia da ferramenta em agrupar mapas conceituais conforme o desempenho cognitivo, realizou-se um experimento com mapas obtidos numa escola municipal, construídos em sala de aula por estudantes de três turmas distintas: sexto ano do Ensino Fundamental (T1), contendo 30 estudantes; nono ano do Ensino Fundamental (T2), contendo 18 estudantes; e terceiro ano do Ensino Médio (T3), contendo 18 estudantes. A disparidade de nível das turmas foi utilizada como critério de seleção.

Os mapas foram construídos com lápis e papel, sob orientação do aplicador e apoio do professor responsável pela turma. Antes disso, o aplicador proporcionou: (i) explicação oral sobre o experimento; (ii) explicação e exemplificação sobre mapas conceituais e suas características; (iii) definição de regras para a construção do mapa, a fim de conterem basicamente substantivos como conceitos e verbos como ligações. Em seguida, cada estudante recebeu um mesmo texto de trezentas palavras sobre o tema "mapas conceituais" a ser lido antes da construção do mapa conceitual individual. Ao final de uma hora, os mapas construídos pelos estudantes foram recolhidos.

Posteriormente, o aplicador do experimento fez uma análise do material produzido. Nessa etapa, observaram-se alguns pontos críticos: havia proposições inteiras, datas ou nomes próprios no lugar dos conceitos; relações definidas apenas por preposições, sem verbo; conceitos ligados diretamente a outros conceitos sem rótulo de relação; conceitos duplicados; inexistência de setas orientando a direção da relação entre conceitos, entre outros. Com essas características, foi facilmente percebido o desvio do roteiro proposto para a tarefa, principalmente pelos alunos da $\mathrm{T} 1$, o que pode ser justificado pelo fato de os estudantes não terem tido contato com mapas conceituais anteriormente. Assim, para garantir a continuidade do experimento, apenas dez mapas de cada turma foram escolhidos pelo aplicador como fonte de dados, utilizando como critério a qualidade estrutural. Ou seja, foram selecionados mapas que respeitavam as regras definidas pelo aplicador (substantivos como conceitos e verbos como ligações). A Figura 2 apresenta três dos mapas conceituais construídos pelos aprendizes.

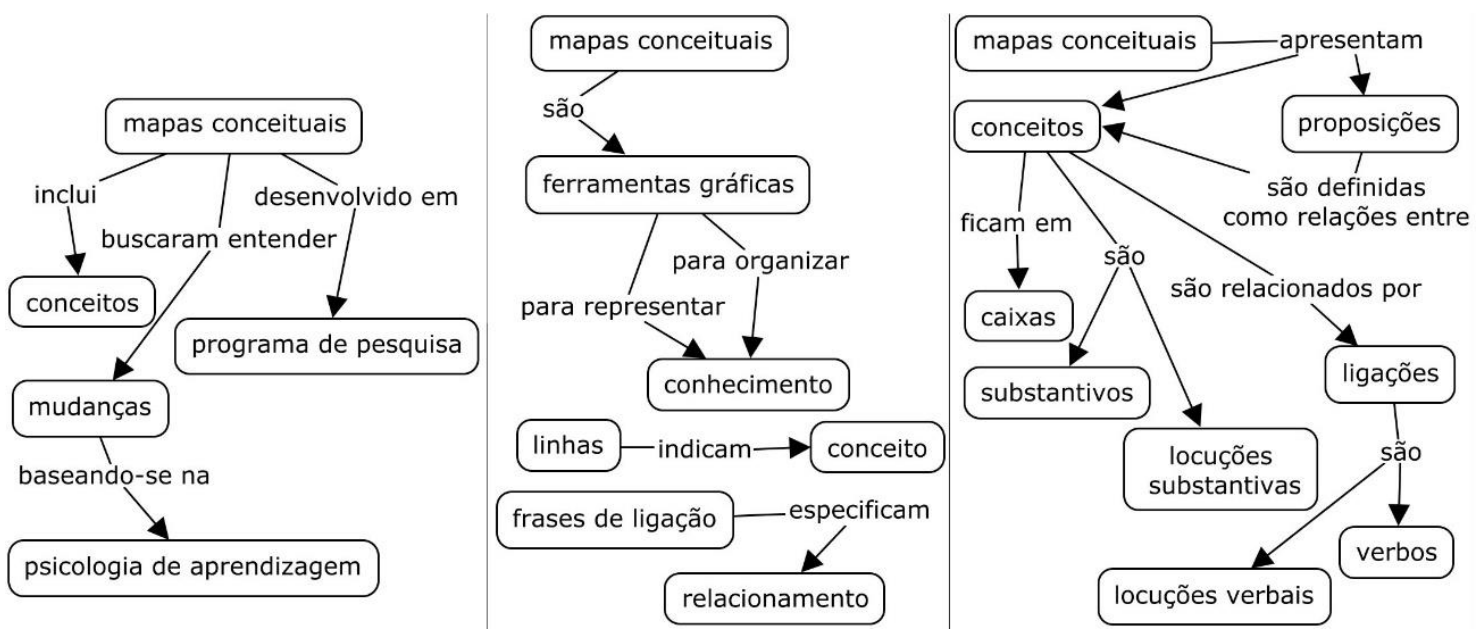

Figura 2. Exemplos de mapas conceituais construídos pelas turmas T1, T2 e T3, respectivamente

Cada mapa foi transcrito para um arquivo de extensão .cxl, formato adotado pela 
ferramenta CmapTools. Através do módulo de extração de informações, os conceitos, pares de conceitos relacionados e proposições foram extraídos a partir dos arquivos e salvos numa base como n-gramas a serem processados. $\mathrm{O}$ módulo de processamento textual realizou a etapa de normalização, removendo as stopwords, identificando a classe de cada palavra e realizando a lematização. Na etapa de redução de variação, a transformação de voz passiva para ativa foi realizada corretamente em poucos casos, o que ressaltou a necessidade de aperfeiçoamento das regras. De forma geral, o módulo de processamento textual apresentou bons resultados, mas sua acurácia precisa ser verificada estatisticamente e aprimorada para as execuções futuras.

A Tabela 1 apresenta o conjunto de n-gramas extraído a partir do mapa de exemplo da turma T3, ilustrado na Figura 2, após o processamento textual.

Tabela 1. Conjunto de n-gramas obtido a partir do mapa da turma T3

\begin{tabular}{|l|l|l|}
\hline N-gramas conceito & N-gramas conceito-conceito & N-gramas conceito-relação-conceito \\
\hline mapa conceitual & mapa conceitual-conceito & mapa conceitual-apresentar-conceito \\
proposicao & mapa conceitual-proposicao & mapa conceitual-apresentar-proposicao \\
conceito & conceito-caixa & conceito-ficar-caixa \\
caixa & conceito-substantivo & conceito-ser-substantivo \\
substantivo & conceito-locucao substantiva & conceito-ser-locucao substantiva \\
locucao substantiva & ligacao-conceito & ligacao-relacionar-conceito \\
ligacao & ligacao-verbo & ligacao-ser-verbo \\
verbo & ligacao-locucao verbal & ligacao-ser-locucao verbal \\
locucao verbal & conceito-proposicao & conceito-definir-proposicao \\
\hline
\end{tabular}

Tomando o conjunto de n-gramas de cada mapa conceitual, o módulo de clusterização representou cada um deles como vetores utilizando a ponderação booleana. A partir dos vetores, o algoritmo K-Means foi executado com as quantidades de clusters possíveis de serem formadas. Como no experimento o número de mapas é igual a 30, foram testadas as quantidades de clusters iniciando de 2 até 29, conforme a regra especificada na Subseção 4.3. A Tabela 2 apresenta as quantidades de clusters que obtiveram melhor média de escore de silhueta e sua respectiva medida de inércia. Como pode ser visto, a execução com parâmetro de quantidade de clusters igual a três obteve melhor média de escore de silhueta. Ou seja, de acordo com a ferramenta, a melhor divisão dos mapas seria feita considerando três grupos, o que condiz com a fonte de dados utilizada (mapas de três turmas distintas).

Tabela 2. Métricas obtidas a partir das execuções do algoritmo K-Means

\begin{tabular}{|l|c|c|c|c|c|c|c|c|c|}
\hline $\mathrm{N}^{\text {o de clusters }}$ & 2 & $\mathbf{3}$ & 4 & 5 & 6 & 7 & 8 & 9 & 12 \\
\hline Média escore silhueta & 0.40 & $\mathbf{0 . 4 7}$ & 0.34 & 0.37 & 0.31 & 0.29 & 0.28 & 0.21 & 0.19 \\
\hline Inércia & 186.52 & $\mathbf{1 0 8 . 2 2}$ & 96.35 & 83.97 & 74.25 & 69.02 & 61.09 & 54.15 & 41.40 \\
\hline
\end{tabular}

Analisando a divisão em três clusters realizada pelo algoritmo, observou-se que 27 dos 30 mapas (90\%) foram colocados no grupo considerado mais apropriado, ou seja, junto dos demais construídos pela mesma turma. Isso significa que a ferramenta conseguiu identificar os três diferentes níveis de desempenho cognitivo representados pelas três turmas. As exceções encontradas podem ser justificadas considerando que numa turma possa haver alunos com nível de desenvolvimento que se assemelha mais ao encontrado em outras turmas. 
Embora o experimento não tenha incorporado o papel do professor, o módulo do mediador apresentou a divisão dos estudantes em três grupos em primeiro lugar na lista, demonstrando que essa seria a mais adequada. A partir dela, seria possível realizar a organização de grupos homogêneos de alunos para fornecimento de material instrucional, bem como a organização de grupos heterogêneos para realização de trabalhos colaborativos.

\section{Conclusões e Trabalhos Futuros}

A partir do trabalho realizado, pode ser reafirmada a necessidade da utilização de ferramentas que possibilitem uma melhor organização de grupos de aprendizes para a realização de trabalhos colaborativos, dada a importância do trabalho em grupo para a construção do conhecimento e a dificuldade de organizar grupos de forma manual. Para tanto, foi proposta uma ferramenta que utiliza a clusterização de mapas conceituais como forma de identificar aprendizes com desempenho cognitivo semelhante e posteriormente sugerir a formação de grupos homogêneos ou heterogêneos em desempenho cognitivo de acordo com a estratégia pedagógica planejada.

Considerando que a construção do conhecimento envolve constantes adições e reformulações, entende-se que a configuração dos grupos deverá sofrer alterações durante o curso. Por isso, a ferramenta proposta deve ser executada várias vezes e com versões atualizadas de mapas conceituais, de modo a propor a melhor organização com base no desempenho cognitivo atualizado dos aprendizes.

A correta execução da ferramenta e a obtenção de resultados que refletem o desempenho cognitivo dos aprendizes dependem fortemente da qualidade estrutural dos mapas, como foi abordado na seção anterior. Por isso, a principal ameaça à validade desse trabalho é o fornecimento de mapas conceituais à ferramenta que não respeitam as regras estruturais apontadas, ainda que contenham proposições válidas. Para diminuir a possibilidade de ocorrência desse problema, é desejável que os aprendizes já estejam previamente familiarizados com mapas conceituais, a fim de que consigam construí-los corretamente.

Os trabalhos futuros estão relacionados ao aperfeiçoamento da proposta desenvolvida: aplicar uma validação automática sobre os mapas antes do processamento realizado pelo módulo de extração de informações e revisar as regras utilizadas na redução de variação feita no módulo de processamento textual para identificação correta de proposições na voz passiva. Ademais, duas questões de grande relevância para a melhoria na qualidade da clusterização serão (i) definir melhor estratégia de pesagem de cada tipo de n-grama utilizado na vetorização dos mapas e (ii) escolher a métrica mais adequada a ser usada para cálculo da similaridade entre os mapas.

\section{Referências}

Cañas, A. J., Novak, J. D., Miller, N. L., Collado, C. M., Rodríguez, M. e Concepción, M. (2006) "Confiabilidad de una Taxonomía Topológica para Mapas Conceptuales". Concept Maps: Theory, Methodology, Technology. v.1, p. 153-161. San Jose.

Davis, C., Silva, M. A. S. e Esposito, Y. L. (1989) "Papel e valor das interações na sala 
VI Congresso Brasileiro de Informática na Educação (CBIE 2017)

Anais do XXVIII Simpósio Brasileiro de Informática na Educação (SBIE 2017)

de aula”. Cadernos de pesquisa. v.71, p. 49-54. São Paulo.

Jain, A. K. (2010) "Data clustering: 50 years beyond K-means". Pattern Recognition Letters. v.31, p. 651-666. Nova Iorque: Elsevier.

Kardan, A. A., Hendijanifard, F. e Abbaspour, S. (2009) "Ranking Concept Maps and Tags to Differentiate the Subject Experts in a Collaborative E-Learning Environment". In 4th International Conference on Virtual Learning. Bucareste.

Kinchin, I. M. e Hay, D. B. (2004) "Using concept maps to optimize the composition of collaborative student groups: a pilot study". Issues and Innovations in Nursing Education. v.51, p. 182-187. Londres.

Mintzes, J. J., Wandersee, J. H. e Novak, J. D. (2000) "Assessing science understanding: A human constructivist view”. San Diego: Academic Press.

Moreira, M. A. (1984) "O mapa conceitual como instrumento de avaliação da aprendizagem”. Instituto de Física da Universidade do Rio Grande do Sul.

Moro, M. L. F. (1991) "Crianças com crianças aprendendo: interação social e construção cognitiva”. Cadernos de Pesquisa. v.79, p. 31-43. São Paulo.

Novak, J. D. (1977) “A Theory of education”. Nova Iorque: Cornell University Press.

Novak, J. D. e Cañas, A. J. (2010) "A teoria subjacente aos mapas conceituais e como elaborá-los e usá-los”. Práxis Educativa. v.5, p. 9-29. Ponta Grossa.

Ratnaparkhi, A. (1996) “A Maximum Entropy Part-Of-Speech Tagger”. In Proceedings of the Empirical Methods in Natural Language Processing Conference. University of Pennsylvania.

Schmid, H. (1994) "Probabilistic Part-of-Speech Tagging Using Decision Trees". In Proceedings of International Conference on New Methods in Language Processing, Manchester.

Souza, F. P., Ciarelli, P. M. e Oliveira, E. (2014) "Combinando Fatores de Ponderação para Melhorar a Classificação de Textos". Anais do Computer on the Beach 2014, p. 32-41. Itajaí.

Valerio, A., Leake, D. B. e Cañas, A. J. (2008) "Automatic classification of concept maps based on a topological taxonomy and its application to studying features of human-built maps". In Proceedings of the 3rd International Conference on Concept Mapping. Tallin.

Vygotsky, L.S. (2007) "Interação entre aprendizado e desenvolvimento". A formação social da mente. cap. 6. $7^{\mathrm{a}}$ ed. São Paulo: Martins Fontes.

Wagstaff, K., Cardie, C., Rogers, S. e Shroedl, S. (2001) "Constrained Kmeans Clustering with Background Knowledge". In Proceedings of the Eighteenth International Conference on Machine Learning, p. 577-584. Palo Alto.

Wood, D. e O’Malley, C. (1996) “Collaborative learning between peers: an overview”. Educational Psychology in Practice. v.11, p. 4-9. 